\title{
Collaborative care management improves physical functioning in older people with depression
}

Callahan CM, Kroenke K, Counsell SR, et al. IMPACT Investigators. Treatment of depression improves physical functioning in older adults. J Am Geriatr Soc 2005;53:367-73.

Does collaborative care management improve physical functioning in older people with depression?

METHODS

Design: Randomised controlled trial.
Sollow up period: Twelve months.
1999 to 2001 .
People: 1801 people aged over 60 years with current major
depressive or dysthmic disorder (DSM-IV).
Interventions: Improving Mood: Providing Access to
Collaborative Treatment (IMPACT) intervention or usual care.
IMPACT collaborative care management consisted of participant
education on late life depression (videotape and booklet) and
12 months' access to a depression clinical specialist who
coordinated and monitored care with the participant's primary
care physician, a liaison primary care expert, and a psychiatrist.
Treatment was provided according to an approved algorithm.
Usual care consisted of access to primary care and speciality
mental health services.
Outcomes: Physical functioning (instrumental activities of daily
living (IADL; range 0-7) and physical component summary of the
12 item Short Form (PCS; range 0-100) scores). Higher PCS and
lower IADL scores indicate better physical function.
Patient follow up: $96 \%$ at 12 months' follow up.

For correspondence: Christopher $M$ Callahan, $M{ }^{\prime}$ D, Indiana University Center for Aging Research, Regenstrief Institute Inc, 1050 Wishard Boulevard, RG6, Indianapolis, IN 46202, USA; ccallaha@iupui.edu

Sources of funding: John A Hartford Foundation, the California Healthcare Foundation, the Hogg Foundation, and the Robert Wood Johnson Foundation.

\section{MAIN RESULTS}

Collaborative care management significantly improved physical functioning compared with usual care at 12 months (mean PCS scores: 40.91 with collaborative care management $v 39.17$ with usual care, $\mathrm{p}<0.001$; mean IADL scores: 0.73 with collaborative care management $v 0.89$ with usual care, $\mathrm{p}=0.04$ ).

\section{CONCLUSIONS}

Collaborative care management improves physical functioning compared with usual care in older people with depression.

\section{Commentary}

Tr he Improving Mood: Providing Access to Collaborative Treatment (IMPACT) reported by Callahan et al addresses an important question: how can we improve or at least maintain the level of physical functioning in older depressed adults? Although the parallel between decline in physical functioning and worsening of depression has been established by many studies, there have been few studies of interventions aimed at attenuating this decline.

The large sample size and inclusion of people with comorbidities in the IMPACT study make the study population more likely to be a representative of older depressed adults. However, one limitation is that more than three quarters of the sample was white, which may affect the generalisability of the findings. Another issue of concern identified by the authors, is how to interpret the improvement of 1.7 points on the 100point Short Form physical functioning scale seen with the intervention. Although the improvement is statistically significant, whether it is clinically significant is unclear. Furthermore, discerning whether the effect seen can be attributed to a particular component of the intervention, and the extent to which the effect on physical function is dependent on improvement of depression requires further investigation.

Callahan et al show that treatment of depression among older adults can improve or at least maintain physical functioning. Implementing such a collaborative care management model across primary care may increase the independence of older adults with depression and reduce the strain on family members and healthcare providers.

Martine A Buist-Bouwman, MA

University Medical Center Groningen, Groningen, the Netherlands

1 Unutzer J, Katon W, Callahan CM, et al. Collaborative care management of late-life depression in the primary care setting. JAMA 2002;288:2836-45. 